\title{
Co-registration of Bone CT and SPECT Images Using Mutual Information
}

\author{
Lisa Tang*, Ghassan Hamarneh*, and Anna Celler ${ }^{\dagger}$ \\ * Medical Image Analysis Lab, School of Computing Science, Simon Fraser University \\ ${ }^{\dagger}$ Medical Imaging Research Group, Department of Radiology, University of British Columbia
}

\begin{abstract}
We present an automatic and accurate technique for $3 D$ co-registration of SPECT and CT. The method will allow for attenuation correction of SPECT images and fusion of the anatomic details from $C T$ and the functional information from SPECT. Registration was achieved by optimizing the mutual information metric over the parameter space defined by the translation and rotation parameters. To improve the robustness and accuracy of the algorithm, registration was performed in a coarse-to-fine manner. We applied the algorithm on three clinical data sets originating from 1 pelvic and 2 thoracic studies. Validation was done by inspecting the $2 D$ and $3 D$ fusion of the registered images and by observing the convergence in the metric and the transformation parameters. We also evaluated quantitatively the effects of the choice of the parameters, the number of multiresolution levels, and initial misalignment of the paired volumes. Registration of both studies converged close to a final alignment with a maximum translational error of $1.41 \mathrm{~mm} \pm 0.78 \mathrm{~mm}$ and rotational error of $1.21^{\circ} \pm 0.46^{\circ}$ for the thoracic study and a maximum translational error of $1.96 \mathrm{~mm} \pm 1.27 \mathrm{~mm}$ and rotational error of $0.57^{\circ} \pm 0.34^{\circ}$ for the pelvic studies. The average computation time on a $3.0 \mathrm{GHz}$ $P C$ was $<4$ minutes for the entire registration procedure. We conclude that the algorithm had successfully co-registered the CT and SPECT images.
\end{abstract}

\section{INTRODUCTION}

Single photon emission computed tomography (SPECT) is a functional diagnostic technique that uses the detection of photons emitted by the radiotracer in the patient body to create a 3-dimensional image of this tracer distribution. Examples of its clinical applications include studies of blood perfusion in the brain and lungs; assessment of cardiac function, evaluation of myocardial infarcts and coronary artery diseases; and primary and metastatic tumor detection [1]. Additionally, thanks to SPECT's ability to quantify radionuclide-tissue concentration, it also aids radiotherapy planning for different types of malignancies [2], [3].

SPECT images, however, often lack anatomical features, making localization of regions of interests difficult [4]. Furthermore, its diagnostic and quantitative accuracy is adversely affected by photon attenuation and scatter [6], [7]. Collimator blurring also causes images to have relatively poor resolution.

On the other hand, computed-tomography (CT) images capture information about the density distribution in the body and thus, can show several types of tissue with great clarity and good resolution. Unfortunately, CT images alone are often insufficient for most diagnoses, as they are sensitive only to differences in densities. This may lead to problems with differentiating living from necrotic tissues and tumor from scar [4], as well as problems with identifying regions of altered functionality.

In many situations, the diagnostic decisions may be substantially improved if the information from both SPECT and CT

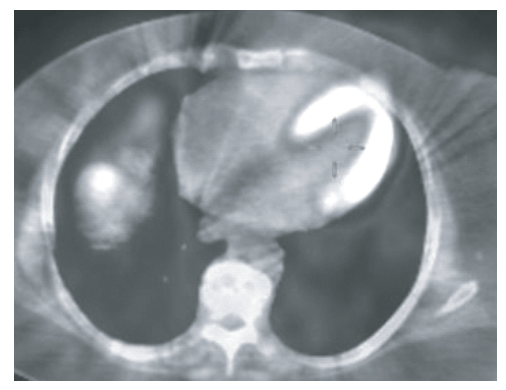

Fig. 1. An example of a cardiac SPECT image acquired by a SPECT-CT hybrid system shown in transaxial view. Misregistration is clearly visible in the apex regions of the heart.

images is combined. Good resolution, anatomical CT images can help in localizing regions that are identified as disease sites in SPECT functional analysis. In addition, since CT images are inherently sensitive to tissue density distribution, they can be used as attenuation maps, which are necessary for SPECT attenuation and scatter correction. Furthermore, SPECT quantitation and its poor resolution may be improved by incorporating CT-based information about organ boundaries into the image reconstruction procedures [10].

All these reasons call for the registration of CT and SPECT images to bring these images into spatial alignment. After registration, fusion of SPECT and CT images can be correctly performed to combine the functional information from SPECT with the anatomical information from CT. This fusion can yield more accurate delineation of tumor compared to CT alone and hence, can be used to guide radiation treatment planning [4]. In addition, by applying the transformation determined in the registration process to the attenuation coefficient maps obtained from the CT images, attenuation correction can also be easily performed on the SPECT images [7].

Registration, in fact, is needed not only for retrospective studies that are performed at different times and using separate SPECT and CT systems. Despite of the development of hybrid SPECT-CT and PET-CT systems, which, in principle, should allow for simultaneous emission-transmission acquisitions [8], these systems still perform sequential scans so the acquired images can easily be misregistered if the patient breathes or moves between acquisitions [4], [5], [11] (see Fig. 1). Therefore, a software-based automatic registration technique is still highly desirable.

Many methods exist for general registration problems. For multi-modality image registration, however, these methods are mostly feature-based or intensity-based [9]. In featurebased algorithms, corresponding anatomic landmarks, organ 
TABLE I

DATA SET SPECIFICATIONS

\begin{tabular}{|l|l|l|l|}
\hline Study Type & Image & Resolution & Voxel size \\
\hline Pelvic & CT & $512 \times 512 \times 109$ & $0.977 \times 0.977 \times 3.0$ \\
& SPECT & $128 \times 128 \times 128$ & $4.664^{3}$ \\
\hline Thoracic & CT & $512 \times 512 \times 125$ & $0.650 \times 0.650 \times 3.0$ \\
& SPECT & $128 \times 128 \times 70$ & $4.664^{3}$ \\
\hline
\end{tabular}

surfaces, or other features are aligned. Although these methods are efficient, they often require significant user interaction and their success highly depends on the accuracy of image segmentation. Conversely, intensity-based methods, which are typically more computationally intensive, may be more robust because they do not rely on segmentation results. And because these methods use all information contained in the images, they may be more appropriate for multi-modal applications.

In recent years, the use of mutual information (MI) as the similarity measure has been proven versatile and successful for multi-modal applications. Some of these include registering CT with PET images of the brain [20]; MRI with PET images of the brain [24]; CT with whole-body PET images of the thorax [13], [21]; and CT-MRI images of the pelvis [12]. A problem with this measure is that it requires that one image provides most information about the other image when they are aligned [20]. This condition is not necessarily true in most studies. In liver studies, for instance, the accumulation of radioactivity in the liver is so high such that other organs are hardly discernible in the SPECT image [18]. Consequently, while the corresponding CT image contains sufficient amount of anatomical information, the overall mutual information is still minimal and so registration often fails [19].

A solution to this problem may be to limit the application of registration to images with similar characteristics and/or images that would produce high MI measure. As an example of this situation, in this paper, we propose to co-register bone SPECT images with CT images. Because bones are clearly visible in both images, we hypothesize that they would yield high amount of mutual information to increase the chance of success of registrations.

In this paper, we first describe the materials in Section II and methods in Section III. Next, we present our registration results in Section IV and findings in Section V. Finally, we make our conclusions in Section VI.

\section{MATERIALS}

Three pairs of CT and SPECT images selected from a clinical database were used to evaluate the registration algorithm. Two pairs came from bone pelvic studies and one from a thoracic study. Details on each data pair are listed in Table I. Before input to the registration algorithm, each CT volume was resampled to an isotropic volume with slice matrix of $256 \times 256$.

\section{MethodS}

\section{A. Mutual information}

According to information theory, the amount of information an image contains is measured by its entropy. For a given image $A$, its entropy is defined as

$$
H(A)=\sum_{i} p(i) \log p(i)
$$

where $i$ is the intensity values in $A$ and $p(i)$ is the marginal probability distribution function (PDF) of $i$. Similarly, the amount of combined information of two images is measured by their joint entropy. Given two images $A$ and $B$, their joint entropy is

$$
H(A, B)=\sum_{i, j} p(i, j) \log p(i, j)
$$

where $i$ is the PDF of $A$ and $j$ is the PDF of $B$. With these definitions, the mutual information of $A$ and $B$ can be defined as

$$
M I(A, B)=H(A)+H(B)-H(A, B) .
$$

In essence, the mutual information of $A$ and $B$ tells us how well $A$ explains $B$ [17]. If we think of entropy as a measure of uncertainty in the image, then mutual information is the amount by which the uncertainty about $B$ decreases when $A$ is given.

The task of registration can be thought of as the process of minimizing $H(A, B)$. This can be visualized by observing the joint histogram that is constructed by plotting the frequencies of occurrence for each intensity pair that occur at the same location. When two images are misaligned, the number of different intensity pairs is high and hence their joint entropy is high. This leads to high dispersion in the joint histogram [16]. In contrast, when the images are well registered, their joint entropy is low and hence the dispersion in the joint histogram is low as well. However, because $H(A, B)$ is highly sensitive to the contents of the overlapping regions of $A$ and $B$, it is possible that it has a low value even when $A$ and $B$ are completely misregistered. In fact, this occurs when only their background regions overlap. To compensate for this limitation, the entropies of $A$ and $B$ are included in the formulation of mutual information so that if the overlapping parts of the images contain only background intensity values, then $H(A)+H(B)$ will be low, leading to an overall low MI value [15].

\section{B. Implementation and optimization algorithm}

The registration framework provided by the Insight Toolkit (ITK) library was used in this study. This framework provides various pluggable components that can be interchanged such that different pipelines can be designed to suit different registration applications [14]. The registration pipeline that we use is depicted in Fig. 2.

In this study, we used the implementation by Mattes et al. [22] to calculate the MI value. This implementation draws one set of samples from the fixed image. The marginal and joint PDFs are then constructed at discrete positions or bins 


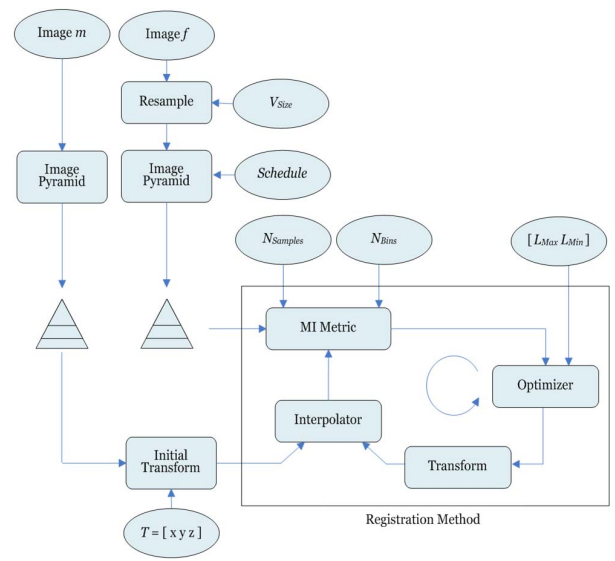

Fig. 2. The basic components of our multi-resolution registration pipeline. The fixed image $f$ is first resampled to an isotropic volume of voxel size $V_{\text {size. }}$ Two pyramids are then constructed from the resampled image $f$ and the moving image $m$ according to Schedule, which encapsulates the scaling factor $S_{x}, S_{y}, S_{z}$ in x, y, z dimensions respectively. Next, an initial alignment $T$ is applied to the moving image $m$ before registration begins During registration, the metric parameterized by number of bins $N_{\text {bins }}$ and sample size $N_{\text {samples }}$ is optimized with a step size that is within the range of $\left[L_{\min }, L_{\max }\right]$.

uniformly spread within the range of the intensity values [14]. A B-spline kernel is used to calculate the PDF's of the images. Because the PDF of the fixed images does not contribute to the metric derivative, it does not need to be smooth and so a zero order kernel is used. Conversely, the PDF of the moving image contributes to the metric derivative so a third order kernel is used. Note that because a B-spline kernel is used, each intensity sample only affects a small number of bins and hence does not require $O\left(n^{2}\right)$ operations to calculate the metric value [14]. Further, because the image intensity values are linearly scaled to the range of $[0,1]$, no preprocessing is needed to rescale the images, a task that is required in the implementation by Wells et al. [20].

During registration, the optimizer seeks to find the transformation parameters that minimize the negative value of $M I(A, B)$. In each iteration of the registration process, the optimizer takes a step of size $=L_{\max }$ along the direction of the metric derivative. When the direction of the metric derivative changes, the optimizer assumes that it has passed a local extrema and reduces its step size by a half. At the end of each iteration, the moving image is interpolated using linear interpolation to resample voxels that are located at nonintegral coordinates. This process is repeated until the next calculated step size is smaller than $L_{\min }$ or when the number of maximum iterations has been exceeded.

In order to improve the speed, accuracy and robustness of the registration algorithm, we used a multi-resolution approach in which registration is performed using a coarse-to-fine scale. For a multi-resolution of $N_{\text {levels }}$ levels, the scale is defined by $k=N_{\text {levels }}$ sets of shrink factors $\left\{S_{x}, S_{y}, S_{z}\right\}$ at which the images are down-sampled in each dimension. In generating these $k$ images, the original image is first smoothed by a Gaussian filter with variances of $S_{x} * 0.5, S_{y} * 0.5$, and $S_{z} * 0.5$ in each respective dimension. Subsequently, they are downsampled according to the schedule of shrink factors [14]. When registration begins at the coarsest level, the resampled fixed and moving images that have the lowest resolutions are used. After the first run of registration, the spatial mapping determined at this level is then used to initialize the registration at the next finer level. This strategy helps to increase the capture range and reduces the likelihood of entrapment in local minima [7], [14], [21].

\section{Solving for the transformation parameters}

In this preliminary study, we solved for rigid-transformation only. Future work will include non-rigid transformation as the second step to be performed on the rigidly-aligned images. Since the fields of view of the CT and SPECT images of the same patient were defined, scaling was not required, leaving 3 translations $\left(T_{x}, T_{y}, T_{z}\right)$ and 3 rotations $\left(\theta_{x}, \theta_{y}, \theta_{z}\right)$ parameters to be solved for.

To simultaneously solve for the 6 transformation parameters, we used the itk: :VersorRigid3Transform provided in ITK. This transform can be seen as a versor transform plus a translation by a vector. A versor is the rotational part of a unit-quaternion and its 3 components are independent. It is the best representation for rotations in $3 \mathrm{D}$ space because sequences of versors allow smooth rotational trajectories and consequently, allow stable optimization [14]. However, since the space formed by a versor is not a vector space, standard gradient descent algorithms are inappropriate. Hence, we used the itk: :VersorRigid3DOptimizer, a variant of the gradient descent that is specifically designed for optimization in the versor space. This optimizer uses versor composition for updating the 3 components of the versor and vector addition for updating the 3 components of the translation.

\section{Selection of parameters}

According to Luis et al. [14], the number of bins $\left(N_{\text {bins }}\right)$ used in the estimation of $M I$ can significantly impact the performance of the optimizer. While they suggested that $N_{\text {bins }}$ $=50$ is sufficient for typical applications, they also commented that good registration trials indeed require an exhaustive search for this parameter. Similarly, the sample size $\left(N_{\text {samples }}\right)$ has similar influence on the metric calculation and the choice should be made with considerations about the content of the image. They suggested that $N_{\text {samples }}$ should equal to $1 \%$ of the total number of voxels for smooth images that contain very few details or equal up to $20 \%$ for highly detailed images. Again, as they have shown in [14], the selection of $N_{\text {samples }}$ requires exhaustive experiments. We hence conducted separate experiments for $N_{\text {bins }}$ and $N_{\text {samples }}$ as a function of different initial positions of the moving image and different optimization settings.

\section{E. Preprocessing of SPECT images}

We observed that both pelvic bone SPECT images contained artifacts that were caused by partial-volume effects. To reduce the potential noise these artifacts may bring to the calculation 
TABLE II

RANDOM TRANSFORMATIONS OF SET A AND B

\begin{tabular}{|l|l|l|l|l|}
\hline Image Set & \multicolumn{4}{|c|}{ Initial Misalignment } \\
& $\theta$ & $T_{x}$ & $T_{y}$ & $T_{z}$ \\
\hline A & \pm 5 & \pm 10 & \pm 10 & \pm 10 \\
B & \pm 10 & \pm 15 & \pm 15 & \pm 15 \\
\hline
\end{tabular}

of the $M I$ metric, we tried to remove these artifacts through masking. Bone structures were first semi-automatically segmented in the SPECT image to create a binary mask using the Amira Software (Mercury Systems Inc.). Voxels that were classified as bone were retained or masked out if otherwise. The masked image was then input to the registration algorithm. Repeated registration trials - each of varying parameters - were compared against those obtained using the original image.

\section{F. Evaluation of registration accuracy and reproducibility}

Because the ground truth is unknown in all of our studies, we performed the reproducibility test as was done in [21]. This test establishes the capture ranges of the transformation parameters and tells us the limits of acceptable initial misalignments between the images.

The test was done by running multiple registrations of the same data set with initial positions that were determined randomly. Specifically, two sets of images (see Table III for details) were created from the groundtruth position, which was defined as the mean of the transformation parameters for all successful registrations. A trial was classified as successful if the inverse rotation was within $\pm 2^{\circ}$ and if the inverse translation parameters were within the ranges of $\pm 3 \mathrm{~mm}$, $\pm 3 \mathrm{~mm}$, and $\pm 4 \mathrm{~mm}$ in the $\mathrm{x}-, \mathrm{y}-, \mathrm{z}$ - dimensions. These ranges had been increased by $1 \mathrm{~mm}$ from those presented in the study by Wong et al. [23] in which they validated the registration of brain images. This increase was made to account for the increased difficulty in validating the registration of thoracic and pelvic images.

To quantify the results, an error was defined for each trial as the absolute difference between the transformation parameters and the 'groundtruth' parameters. Then, the mean of these errors were calculated. Each of these trials was also repeated to quantify the influence of masking as well as the choice of the number of multi-resolution levels and the metric's parameters.

\section{RESUlts}

All registrations were performed on a $3.0 \mathrm{GHz}$ PC. On average, the construction of two pairs of 3-level image pyramids, with scaling factors of $S_{x}=S_{y}=S_{z}=3$ took 1.57 minutes, while two pairs of 2-level pyramids with $S_{x}=S_{y}=S_{z}=2$ took 0.345 minutes. Both the thoracic and pelvic studies had similar registration times; on average, a successful registration using images from Set A (Table II) took a maximum of 1.22 and registration using images from Set B took a maximum of 2.81 minutes.

Validation was done quantitatively by the reproducibility test and visually through 2D and 3D image fusions (the latter
TABLE IV

EFFECT OF PARAMETERS ON REGISTRATION ACCURACY

\begin{tabular}{|l|l|l|c|c|}
\hline \multicolumn{3}{|c|}{ Metric's parameters } & Thoracic study & Pelvic studies \\
\hline$N_{\text {bins }}$ & $N_{\text {samples }}$ & Ratio & Success rates $^{1}$ & Success rates \\
\hline 10 & 15000 & 0.00067 & 100 & 90.9 \\
15 & 15000 & 0.0010 & 51.2 & 0 \\
25 & 15000 & 0.0017 & 42.7 & 100 \\
\hline 15 & 10000 & 0.0015 & 0 & 100 \\
20 & 10000 & 0.0020 & 0 & 100 \\
25 & 10000 & 0.0025 & 0 & 82.4 \\
\hline
\end{tabular}

${ }^{1}$ Expressed as percentages.

being particularly useful in detecting small rotational errors). Fig. 3 and 4 show examples of these fusions.

In the reproducibility test as described in Section III, we found that registration with 3 levels of resolution had the highest success rates while resolution of fewer than 2 levels or more than 4 levels had slightly lower success rates. The success rates, mean and standard deviation errors of all these tests for each set are summarized in Table III.

The sensitivity of the registration results to the parameters used is summarized in Table IV. From our experiments, when the $N_{\text {samples }}: N_{\text {bins }}$ ratio exceeded 0.00166 , registration of the thoracic images performed poorly in the reproducibility test.

Finally, our experiments also showed that registration with the masked SPECT image performed slightly worst and yield a $83 \%$ success rate. This was mostly due to the fact that the amount of information had been reduced and so the metric became very sensitive to the registration setup and parameters used. However, when a different set of parameters was used (i.e. fewer bins and a smaller sample size), the success rate would increase to $88 \%$.

\section{Discussions}

In general, the effect of the optimization settings on the registration success rates was minimal. On the other hand, the metric's parameters as well as the multi-resolution strategy used had significant influence on the registration result. Unfortunately, finding the right choice for these parameters is not trivial. For instance, the smoothness of the metric calculation is affected if too few samples are taken. However, using more samples does not necessarily lead to smoother calculation but will require more time to collect the samples than the optimal number requires (see Fig. 5). In fact, the selections for the sample size and the number of bins are highly dependent. If we use more bins, we should also increase the sample size or the joint histogram would become too sparse for the metric to operate properly. Consequently, different pairs of $N_{\text {bins }}$, $N_{\text {samples }}$ resulted in very different success rates and error ranges in the transformation parameters.

From our preliminary study, we found no simple rules for determining these parameters. We also did not find any trends of success rates base on these parameters. That is, a pair of $N_{\text {bins }}, N_{\text {samples }}$ that yield a high success rate for the 


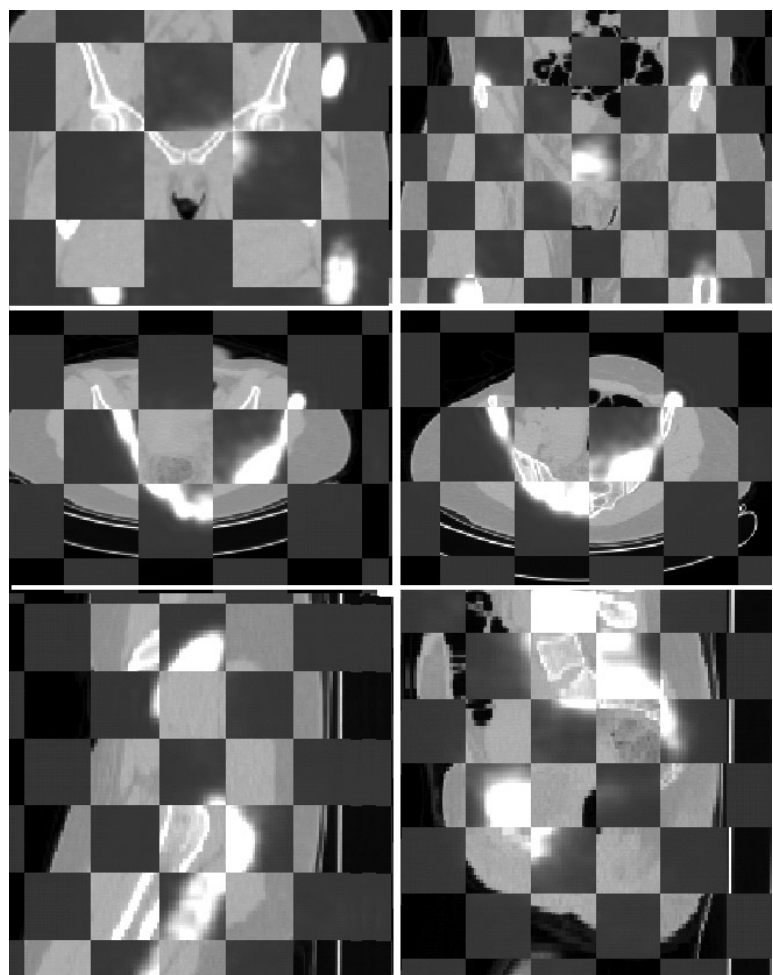

(a)
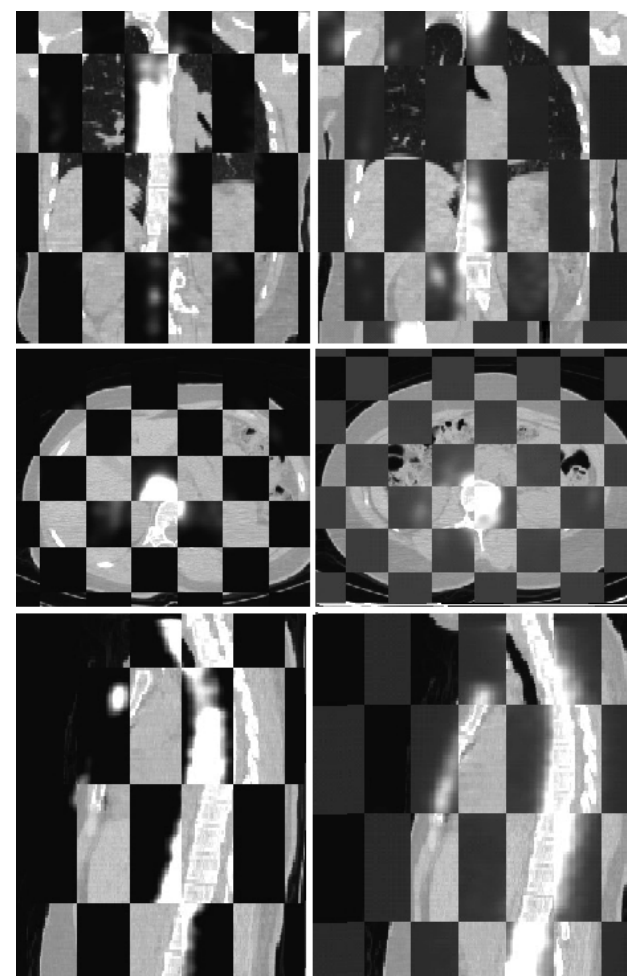

(b)

Fig. 3. Checkerboard composites of the CT and SPECT images before and after registration. (a) Images from one of the pelvic studies before and after registration. (b) Images from the thoracic study before and after registration.

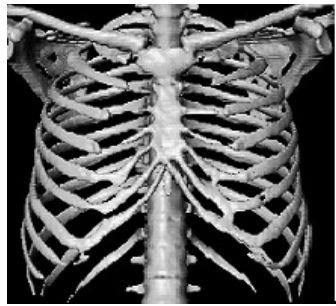

(a)

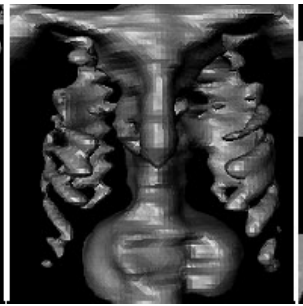

(b)

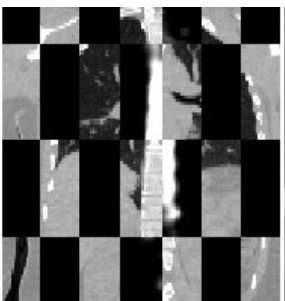

(c)

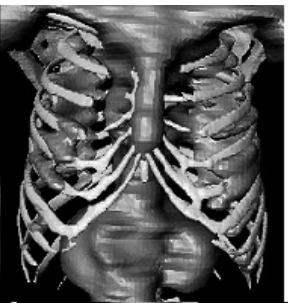

(d)

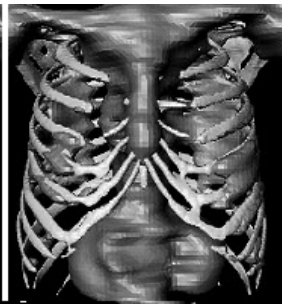

(e)

Fig. 4. To generate 3D fusions, surfaces were extracted from the images to reconstruct 3D surface meshes. (a) The mesh extracted from the CT image in the thoracic study. (b) The mesh extracted from the SPECT image in the same study. (c) the 2D fusion generated after registration. Note that misalignment is not as obvious. (d) The corresponding 3D fusion in which the rotational error is more evident. (e) The 3D fusion of a more successful registration trial.

TABLE III

MEAN AND STANDARD DEVIATION ERRORS OF REGISTRATION TRIALS

\begin{tabular}{|l|l|l|l|l|l|l|l|l|l|l|l|}
\hline Set & No. of & \multicolumn{4}{|c|}{ Success rates $^{1}$ and errors } \\
& Levels & $\%$ & $\theta$ & $T_{x}$ & \multicolumn{4}{|c|}{ Success rates and errors in the thoracic study } \\
& 0 & 63 & $0.41 \pm 0.24$ & $0.92 \pm 0.50$ & $0.54 \pm 0.21$ & $1.18 \pm 0.94$ & 76 & $0.56 \pm 0.43$ & $0.80 \pm 0.78$ & $0.72 \pm 0.57$ & $1.96 \pm 1.27$ \\
& A & 96 & $0.43 \pm 0.25$ & $0.78 \pm 0.42$ & $0.68 \pm 0.50$ & $1.18 \pm 0.81$ & 100 & $0.44 \pm 0.39$ & $0.70 \pm 0.70$ & $0.61 \pm 0.50$ & $1.37 \pm 1.00$ \\
& 2 & 100 & $0.56 \pm 0.39$ & $0.85 \pm 0.63$ & $0.77 \pm 0.48$ & $1.39 \pm 0.83$ & 100 & $0.46 \pm 0.38$ & $0.82 \pm 0.49$ & $1.57 \pm 0.81$ & $1.75 \pm 0.96$ \\
& 3 & 84 & $1.10 \pm 0.57$ & $0.94 \pm 0.64$ & $1.37 \pm 0.71$ & $1.17 \pm 0.83$ & 68 & $0.57 \pm 0.34$ & $0.92 \pm 0.60$ & $0.88 \pm 0.86$ & $1.69 \pm 1.05$ \\
& 0 & 76 & $0.96 \pm 0.44$ & $1.03 \pm 0.69$ & $1.41 \pm 0.78$ & $1.34 \pm 0.79$ & 92 & $0.40 \pm 0.25$ & $0.74 \pm 0.60$ & $0.80 \pm 0.62$ & $1.68 \pm 1.13$ \\
& 2 & 3 & $1.21 \pm 0.46$ & $0.94 \pm 0.56$ & $1.55 \pm 0.82$ & $0.98 \pm 0.79$ & 96 & $0.52 \pm 0.26$ & $0.90 \pm 0.59$ & $0.57 \pm 0.56$ & $1.71 \pm 0.96$ \\
\hline
\end{tabular}

${ }^{1}$ A trial was classified successful if the errors were less than $\pm 2^{\circ}$ for rotation and $\pm 3 m m, \pm 3 m m, \pm 4 m m$ for translation.

${ }^{2}$ Units are $\mathrm{mm}$ for translations and degrees for rotations. 


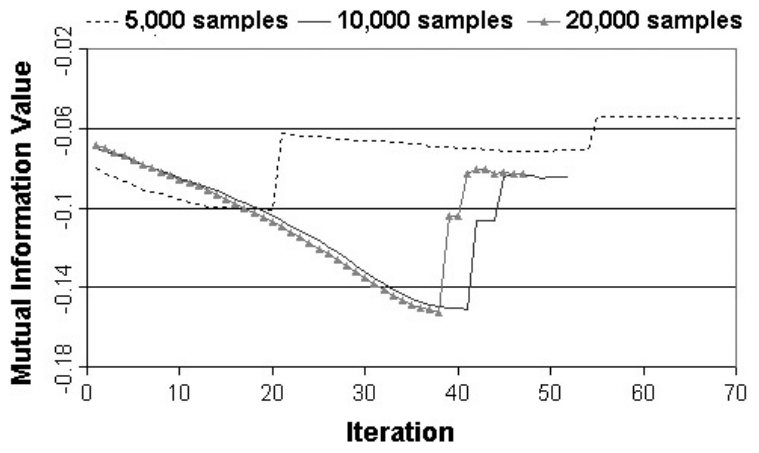

Fig. 5. The evolutions of 3 registration trials on the same data using different sample sizes. Note that using 5,000 samples required more iterations for the metric to converge.

registration of pelvic images may yield a low success rate for the registration of thoracic images, or vice versa. From Table IV, it is evident that the success of the registration of the thoracic images was very sensitive to the parameters chosen.

Similar to other studies, the use of multi-resolution also increased the success rates and decreased the registration error in most of the trials. However, because the original slice thickness of the CT images is $3 \mathrm{~mm}$, which gives an equivalent voxel dimension of $1 \times 1 \times 4.48$, the down-sampling factors $S_{x}, S_{y}, S_{z}$ should be determined accordingly. Specifically, we found that no down-sampling in the z-dimension $\left(S_{z}=1\right)$ and 3 levels of multi-resolution yield the most accurate results.

\section{CONCLUSION}

Registration of multi-modal images is very important for diagnosis, treatment planning, and tumor detection. The registration of CT and SPECT images allow for not only the attenuation correction of SPECT images but also for image fusion that can ease the localization of the regions of interest these images capture.

In this preliminary study, the use of mutual information has shown to be an effective and accurate similarity metric for the registration of CT and SPECT images in thoracic and pelvic studies. Our experiments also show that the success of registration is highly influenced by the selection of parameters. In particular, it is not trivial to determine the sample size and the number of bins for the calculation of the MI metric.

Similar to what many studies have found, the use of multi-resolution in our implementation has also decreased the likelihood of entrapment in local minima. In future, we will expand our current work by including non-rigid transformation to our algorithm to complete the entire registration procedure.

\section{REFERENCES}

[1] A. Webb, Introduction to Biomedical Imaging, Ed. Illinois: IEEE Press, 2003, pp.73-89.

[2] J. C. Roeskea, A. Lujana, R. C. Rebab, B. C. Penneyb, S. D. Yamadac, and A. J. Mundta "Incorporation of SPECT bone marrow imaging into intensity modulated whole-pelvic radiation therapy treatment planning for gynecologic malignancies," Radiotherapy and Oncology, vol. 77, No. 1, pp. 11-17, October 2005.
[3] A. C. Paulino, W. L. Thorstad, and T. Fox, "Role of fusion in radiotherapy treatment planning," Seminars in Nuclear Medicine, vol. 33, No. 3, pp. 238-243, July 2003.

[4] D. E. Heron, R. P. Smith. and R. S. Andrade, "Advances in imageguided radiation therapythe role of PET-CT," Medical Dosimetry, vol. 31, no. 1, pp. 3-11, 2006.

[5] T. M. Blodgett, B. M. McCook, and M. P. Federle, "Positron Emission Tomography/Computed Tomography: Protocol Issues and Options,' Seminars in Nuclear Medicine, vol. 36, no. 2 , April 2006, pp. 157-168.

[6] Y. Masood, Y. H. Liu, G. DePuey, R. Taillefer, L. I. Araujo, S. Allen, D. Delbeke, F. Anstett, A. Peretz, M. J. Zito, V. Tsatkin, and F. J. Wackers, "Clinical validation of SPECT attenuation correction using $\mathrm{x}$ ray computed tomographyderived attenuation maps: Multicenter clinical trial with angiographic correlation," Journal of Nuclear Cardiology, vol. 12, no. 6, pp. 676-687, November 2005.

[7] D. Utsunomiya, S. Tomiguchi, S. Shiraishi, K. Yamada, T. Honda, K. Kawanaka, A. Kojima, K. Awai, and Y. Yamashita, "Initial experience with X-ray CT based attenuation correction in myocardial perfusion SPECT imaging using a combined SPECT/CT system," Annals of Nuclear Medicine, Vol. 19, No. 6, pp. 485489, 2005.

[8] Z. Keidar, O. Israel, and Y. Krausz, "SPECT/CT in tumor imaging: technical aspects and clinical applications," Seminars in Nuclear Medicine, vol. 33, no. 3, pp. 205-218, July 2003.

[9] P. J. Slomka, "Software approach to merging molecular with anatomic information," Journal of Nuclear Medicine, vol. 45, no. 1, pp. 36-45, Jan 2004.

[10] Comtat C., Kinahan, P. E., Fessler, J. A. et al., "Clinically feasible reconstruction of $3 \mathrm{D}$ whole-body $\mathrm{PET} / \mathrm{CT}$ data using blurred anatomical labels," Phys Med Biol 47: 1-20, 2002.

[11] J. Chen, S. F. Caputlu-Wilson, H. Shi, J. R. Galt, T. L. Faber, and E. V. Garcia, "Automated quality control of emission-transmission misalignment for attenuation correction in myocardial perfusion imaging with SPECT-CT systems," Journal of Nuclear Cardiology, vol. 13, no. 1, pp. 43-49, January-February 2006.

[12] R. P. Woods, J. C. Mazziotta, S. R. Cherry, "MRI-PET registration with automated algorithm". J. of Computer Assisted Tomography, vol. 17 pp. 536-546, 1993.

[13] R. Shekhar, V. Walimbe, S. Raja, V. Zagrodsky, M. Kanvinde, G. Wu, and B. Bybel, Automated 3-Dimensional elastic registration of wholebody PET and CT from separate or combined scanners, J. of Nuclear Med., vol. 46, pp. 1488-1496, 2005.

[14] L. Ibanez, W. Schroeder, and the Insight Consortium, The ITK Software Guide, The Insight Consortium, 2005, pp. 347-533. http://www.itk.org/

[15] A. Pluim, J. Maintz, and M. Viergever, "Mutual information based registration of medical images: a survey," in IEEE Trans. Med. Imag., vol. 21, pp. 61-75, 2003.

[16] J. Maintz and M. Viergever, An overview of medical image registration methods, Imaging Science Department, Imaging Center Utrecht, 2000.

[17] D. Hill and D. J. Hawkes, Across-Modality registration using intensitybased similarity metrics, in Handbook of Medical Imaging, I. N. Bankman, Ed. San Diego: Academic Press, 2000, pp. 537-553.

[18] M. Safaraz, X. Wu, M. A. Lodge, and C. X. Yu, ”Automatic CT-SPECT registration of livers treated with radioactive microspheres," Physic. Med. biol., vol. 49, pp.131-137, 2004.

[19] X. Wang, J. Tian, 'Image registration based on maximization of gradient code mutual information," Image Anal. Stereol., vol.24, pp.1-7, 2005.

[20] W. M. Wells, P. Viola, H. Atsumi, S. Nakajima, and R. Kikinis, "Multimodal volume registration by maximization of mutual information," Med. Imag. Analysis, vol. 1, pp. 53-51, 1996.

[21] P. J. Slomka, D. Dey, C. Przetak, U. E. Aladl, and R. P. Baum, "Automated 3-Dimensional registration of stand-alone 18F-FDG wholebody PET with CT,' J. of Nuclear Med., vol. 44, no. 7, pp.1156-1167, July 2003.

[22] D. Mattes, D. R. Haynor, H. Vesselle, and T. Lewellen, "PET-CT image registration in the chest using free-form deformations," IEEE Trans. in Med. Imag., vol. 22, no. 1, pp. 120-128, January 2003.

[23] J. C. Wong, C. StudholmeD. J. Hawkes, and M. N. Maisey, "Evaluation of the limits of visual detection of image misregistration in a brain fluorine-18 fluorodeoxyglucose PET-MRI study," European Journal Nuclear Medicine, vol. 24, pp. 642-650, 1997.

[24] Yokoi, Soma, H. Shinohara, and H. Matsuda, "Accuracy and reproducibility of co-registration techniques based on mutual information and normalized mutual information for MRI and SPECT brain images,' Annals of Nuclear Medicine, vol. 18, no. 8, pp. 657-667, 2004. 